\title{
Review Article \\ Prognostic Histopathological and Molecular Markers on Prostate Cancer Needle-Biopsies: A Review
}

\author{
A. Marije Hoogland, Charlotte F. Kweldam, and Geert J. L. H. van Leenders \\ Department of Pathology, Josephine Nefkens Institute, Room Be-206, Erasmus Medical Center, P.O. Box 2040, \\ 3000 CA Rotterdam, The Netherlands \\ Correspondence should be addressed to A. Marije Hoogland; a.m.hoogland@erasmusmc.nl
}

Received 5 June 2014; Accepted 4 August 2014; Published 27 August 2014

Academic Editor: Andreas Doll

Copyright (C) 2014 A. Marije Hoogland et al. This is an open access article distributed under the Creative Commons Attribution License, which permits unrestricted use, distribution, and reproduction in any medium, provided the original work is properly cited.

\begin{abstract}
Prostate cancer is diverse in clinical presentation, histopathological tumor growth patterns, and survival. Therefore, individual assessment of a tumor's aggressive potential is crucial for clinical decision-making in men with prostate cancer. To date a large number of prognostic markers for prostate cancer have been described, most of them based on radical prostatectomy specimens. However, in order to affect clinical decision-making, validation of respective markers in pretreatment diagnostic needle-biopsies is essential. Here, we discuss established and promising histopathological and molecular parameters in diagnostic needle-biopsies.
\end{abstract}

\section{Introduction}

Prostate cancer is the most common cancer in Western men. Although prostate cancer follows an aggressive course in a significant number of men, most tumors do not cause significant clinical symptoms. Therefore individual assessment of a tumor's aggressive potential is crucial for clinical decisionmaking in men with prostate cancer.

In general, prostate cancer is diagnosed on needle-biopsies prompted by elevated serum prostate specific antigen (PSA) levels, suspicious digital rectal examination or transrectal ultrasonography findings, and/or clinical symptoms of urinary tract obstruction. In addition to elevated PSA levels and clinicoradiological signs of either local extension or metastasis, detailed histopathological characterization of prostate cancer at needle-biopsies predicts clinical tumor behavior and sustains therapeutic decision-making. In daily practice, the pathology report of prostate cancer includes the grade of differentiation according to the modified Gleason grading system, the number of biopsies infiltrated by prostate cancer, and a quantitative assessment of the tumor volume per biopsy in either length in $\mathrm{mm}$ or percentage of tumor $[1,2]$. Implementation of novel histopathological and molecular markers is required for several reasons. While only half of the patients has a potentially life-threatening prostate cancer (Gleason score $\geq 7$ ), 55-90\% of patients with low-risk disease still undergo radical prostatectomy [3]. Active surveillance has become a widely used alternative for treatment after prostate cancer diagnosis. However, up to $33 \%$ of patients on active surveillance need therapeutic intervention after a median follow-up of 1.2-3.5 years [4-8]. Therefore, better stratification of prostate cancer patients with respect to clinical decision-making is necessary, especially in the predominant group of low- to intermediate-risk prostate cancers.

Another reason to implement novel markers in prostate cancer diagnosis and clinical decision-making is the considerable interobserver variability in Gleason grading among pathologists. This interobserver variability is particularly of significance in the large group of low- to intermediate-risk prostate cancer, as it can influence therapeutic approaches [8-10]. Contemporary modified Gleason grading in needlebiopsies demands adding the most common and highest Gleason grade to the final Gleason score, regardless of the amount of "highest" Gleason grade. In practice, when only a few atypical glands considered Gleason grade 4 are present together with a large volume of Gleason grade 3, the Gleason score is 7 and excludes patients from active surveillance in our institute. As considerable interobserver variability exists between the distinction of Gleason grades 3 and 4, important 
treatment decisions depend too much on individual pathologist's opinions. Thus, it is important to improve the reproducibility of Gleason grading by more objective parameters. In particular, molecular markers reflecting tumor biology could act as novel threshold in active surveillance or watchful waiting.

Finally, spatial heterogeneity of prostate cancer might lead to under- or, rarely, overestimation of prostate cancer aggressive potential on diagnostic needle-biopsies. In general, prostate biopsies only sample $0.05 \%$ to $0.5 \%$ of the total prostate volume, which might result in undersampling of the most significant area of prostate cancer tissue. In addition to improved image-guided needle-biopsy procedures, implementation of novel molecular markers might predict the presence of unsampled significant areas in case molecular aberrations precede pathologically discernible patterns.

Last decade, much effort has been put in the identification of novel histopathological and molecular markers to further improve prediction of tumor behavior in prostate cancer patients. The vast majority of research has focused on correlation of novel markers with static clinicopathologic parameters at radical prostatectomy such as Gleason score, pTstage and surgical margin status, or biochemical recurrence after operation. However, in order to affect clinical decisionmaking, validation of respective markers in pretreatment diagnostic needle-biopsies is essential. Here, we discuss established and promising histopathological and molecular tissue markers in diagnostic needle-biopsies.

\section{Pathologic Markers}

2.1. Gleason Grading. The contemporary system for grading prostate cancer was developed by Gleason in the 1960s [11]. The Gleason grading system is solely based on the tumor's architecture. The Gleason score equals the sum of the two most common Gleason grades in radical prostatectomy, and the sum of the most common and highest Gleason grades in needle-biopsies. Up to date, the Gleason score is a strong predictor for disease progression, and one of the most important parameters in therapeutic decision-making.

In 2005, the Gleason grading system was modified at the International Society of Urological Pathology (ISUP) conference [1]. As a result, several tumor growth patterns classically considered as Gleason grade 3 were redefined as Gleason grade 4 [1]. Shortly after, small cribriform and glomeruloid glands have been reconsidered Gleason grade 4 as well [1214]. As a result of this stage migration, Gleason score 7 has become the most common assigned score on prostatic needle-biopsies [15-17]. For instance, Helpap and Egevad showed in 368 needle-biopsy cases a significant change in distribution of modified Gleason score: Gleason score 2-4 decreased from 2.7 to $0 \%$, Gleason score 5 decreased from 2.7 to $0 \%$, Gleason score 6 decreased from 48 to $22 \%$, but Gleason score 7 increased from 26 to $68 \%$ [16]. Generally, modified Gleason grading has improved the predictive value of grading prostate cancer.
2.1.1. Modified Gleason Grading: Up- and Downgrading. The overall concordance of Gleason score between prostate needle-biopsies and radical prostatectomies has improved from $58 \%$ in classic Gleason score to $72 \%$ in modified Gleason score [16]. Uemura et al. found a decrease in downgrading Gleason score in needle-biopsies in comparison to radical prostatectomy ( $15 \%$ versus $20 \%$ ), although overall Gleason score concordance rates did not change significantly [18]. To date, the overall upgrading rate at radical prostatectomy using the modified Gleason score ranges between 26 and $50 \%$ [19-22]. For instance, a large study containing 7643 radical prostatectomies with corresponding needle-biopsies demonstrated that $36 \%$ of cases $(1841 / 5071)$ were upgraded from a needle-biopsy Gleason scores 5-6 to a higher grade at radical prostatectomy [19]. In the same study $72 \%(1143 / 1577)$ had matching Gleason score 7 on biopsy and radical prostatectomy, and Gleason score 8 on biopsy showed a similar distribution for radical prostatectomy Gleason score $4+3=$ 7,8 , and 9-10 [19]. In men with Gleason score 9-10 on needlebiopsy, 58\% (69/119) had a similar Gleason score on radical prostatectomy [19]. Proposed predictors for upgrading are age, high preoperative PSA, larger tumor percentage per core, number of positive cores, presence of perineural invasion, absence of inflammation, and high prostate volume $[19,21]$. On the other hand, predictors for downgrading from any biopsy Gleason score to a lower Gleason score on radical prostatectomy were low preoperative PSA, lower tumor percentage per core, and larger prostate volume on radical prostatectomy $[19,21]$. Although these predictors for up- and downgrading all showed strong statistical significance, the effect on survival was still small. If in one biopsy session, multiple biopsies show differing Gleason scores, for instance, Gleason scores $4+3$ in one biopsy and $3+3$ in another biopsy, also referred to as presence of ComboGS, patients have lower odds of upgrading at time of radical prostatectomy and decreased risk of prostate cancer specific mortality [23, 24].

\subsubsection{Modified Gleason Grading: Correlation with Pathologic} Features at Radical Prostatectomy. The relationship between Gleason score on needle-biopsy and pathological stage on radical prostatectomy has improved since the implementation of the modified Gleason score [15, 25]. For instance, $4315 / 5205$ men $(83 \%)$ with Gleason score 6 on biopsy had organ-confined disease (pT2) at radical prostatectomy, while increasing Gleason score on biopsy was strongly associated with extraprostatic extension (pT3a) and seminal vesicle invasion (pT3b) [25]. Although high pathologic stage is related to positive surgical margins at radical prostatectomy and biochemical recurrence, it should be mentioned that it is not associated with a uniformly poor prognosis [26-28].

\subsubsection{Modified Gleason Grading: Predicting Patient Outcome.} Billis et al. studied the impact of needle-biopsy Gleason score modification on biochemical recurrence free survival. Here, the modified Gleason score was a better predictor for biochemical recurrence than classic Gleason score [15]. Subsequently, the predictive value of the modified Gleason score 
has been validated in other large cohorts $[18,25]$. Uemura et al. showed that the Gleason score was strongly associated with biochemical recurrence, only when the modified Gleason score was applied [18]. Furthermore, in a large study ( $n=$ 7850) from the Johns Hopkins Hospital, Pierorazio et al. correlated biopsy Gleason score with biochemical recurrence. Here, $95 \%$ of the patients with needle-biopsy Gleason score 6 had no biochemical recurrence after 5 years of follow-up [25]. In men with Gleason score $3+4=7$ and Gleason score $4+3=7$ on needle-biopsy the 5 year biochemical recurrence free survival rates were $83 \%$ and $65 \%$, respectively. Men with Gleason score $4+4=8$ or $9-10$ had the lowest 5 year biochemical recurrence free survival rates, $63 \%$ and $35 \%$ respectively. Tollefson et al. indicated that distant metastasis and diseasespecific death are best estimated by a combination of Gleason score, perineural invasion, and Ki-67 expression [29].

Altogether, modified Gleason grading has generally improved the concordance between biopsy and radical prostatectomy Gleason score, associates better with pathologic parameters at radical prostatectomy, and is more predictive for biochemical recurrence as well as metastasis and disease-specific death.

2.2. Tumor Quantification. Currently, the number of positive core biopsies should routinely be mentioned in pathology reports. Additional measures of prostate cancer volume in needle-biopsies better predict disease outcome. Various parameters have been proposed as measure of tumor extent, for example, tumor percentage in single biopsies, tumor length in single biopsies ( $\mathrm{mm}$ ), and number of negative biopsies. These quantitative assignments are required for most clinical nomograms. For instance, the Steyerberg nomogram incorporates number of positive biopsies, total cancer length $(\mathrm{mm})$, and total "normal" tissue length $(\mathrm{mm})$ to predict indolent disease on radical prostatectomy [30]. In addition, most active surveillance protocols are delimitated by the number of positive biopsies and/or a measure of tumor extent per biopsy [31, 32].

One well-studied example of tumor extent is the percentage of cancer in single biopsies, and many studies have confirmed its prognostic value in biopsies followed by radical prostatectomy $[19,25,33-37]$, by dose-escalated external beam radiotherapy [38], or by a combination with hormonal treatment [39]. The location of positive biopsies can additionally be of therapeutic value, for instance for consideration of nerve-sparing surgery. The majority of these studies agree on the predictive value of tumor extent for endpoints such as biochemical recurrence $[19,25,35-38,40]$, metastasis [36-38], and disease-specific death [36, 38]. However, despite its statistical significance, the effect of tumor percentage in biopsies on survival is mostly small. Furthermore, all of the above mentioned publications used different methods to determine the percentage of cancer and different cutoff values. For instance, Vance et al. divided the percentage of cancer in single biopsies into four quartiles $(<2.5 \%,<10 \%$, $<25 \%$, and $\geq 25 \%$ ) [38], while Nelson et al. categorized it in to $0-10 \%, 11-59 \%$, and $60-100 \%$ [36].
Another frequently assessed measure on prostate biopsies is the percentage of positive biopsy cores, defined as the total number of positive cores divided by the total number of biopsy cores obtained. Studies evaluating its prognostic value have validated the independent predictive value for biochemical recurrence free survival [35, 39, 41, 42]. In 2011 Huang et al. analyzed needle-biopsies of 1056 patients treated with external beam radiotherapy and/or hormonal therapy [43]. Using a cutoff value of $>50 \%$ positive biopsy cores they found that percentage of positive biopsy cores is a powerful and independent predictor for distant metastasis free and overall survival [43]. However, when the percentage of positive biopsy cores was adjusted for percentage of cancer in needle-biopsies in another study, it did not provide any additional superior risk stratification for biochemical recurrence, distant metastasis, or disease-specific death [38].

While measuring tumor extent is generally straightforward, minor controversies exist for instance for quantifying discontinuous prostate cancer foci in single biopsies. One could regard separate foci as being part of the same tumor and measure the distance between the outermost foci including intervening normal prostate tissue, or only measure malignant areas without intervening stroma. In this case, recent studies show that discontinuous foci of prostate cancer in needle-biopsies should be regarded and measured as one continuous lesion $[44,45]$.

In short, tumor volume is an important parameter for disease extent, but there is no consensus yet on the best methodology for its assessment. It is clear that assignment of the number of positive biopsies requires identification of separate biopsy cores, even when they are fragmented due to technical procedures. This can be performed by including only one needle-biopsy per cassette, or marking multiple individual cores in one cassette for instance by inking. It is advised that no more than 3 biopsies should be included in one cassette, provided that measures are taken to prevent their curling and floating [46]. The extent of cancer in individual cores is performed by actual measuring of the tumor's length with a ruler or by estimation of tumor percentage by eye-balling. Measuring tumor length is objective and exact, although more time-consuming. If estimation of percentage is applied in daily practice, one should take into account that detection of prostate cancer in short needle-biopsies due to suboptimal technical procedures can result in overestimation of tumor percentage; for instance presence of $2 \mathrm{~mm}$ prostate cancer in a $5 \mathrm{~mm}$ biopsy results in a tumor percentage of $40 \%$, which might erroneously exclude patients from active surveillance.

2.3. Perineural Invasion. The significance of perineural invasion in prostate cancer biopsies remains questionable. In a systematic review Harnden et al. addressed important limitations of 21 studies on perineural invasion in biopsies followed by radical prostatectomy or radiation therapy [47]. First, the presence of nerves in biopsies was not mandatory for patient inclusion. Second, the number of biopsy cores taken and the number of nerves present ranged widely. Third, 


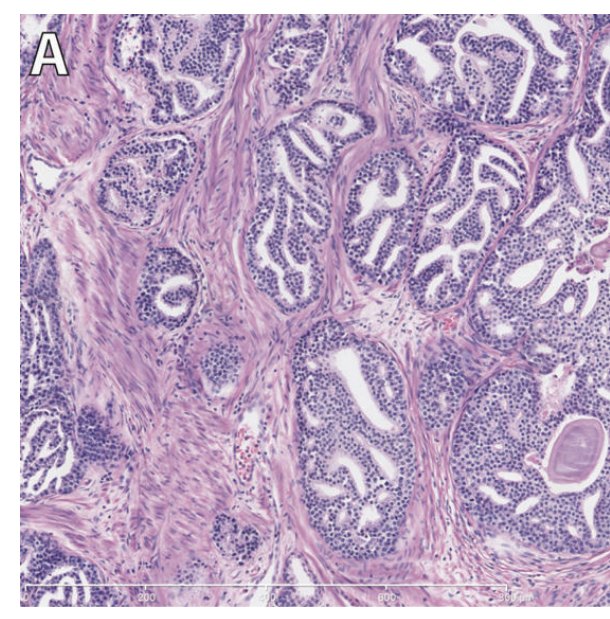

(a)

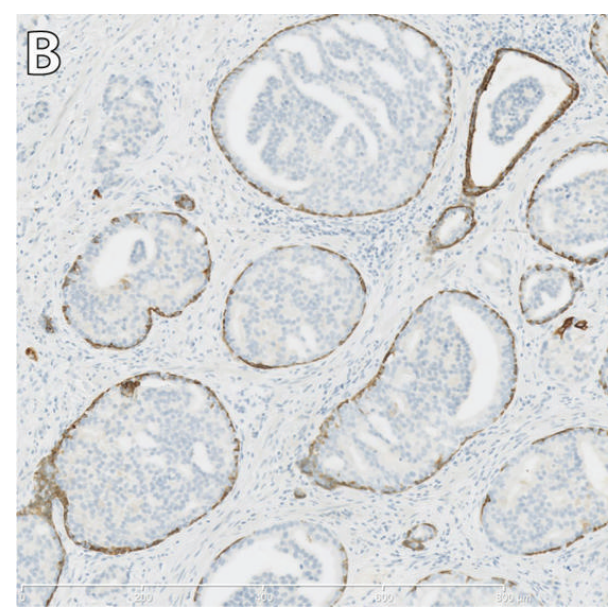

(b)

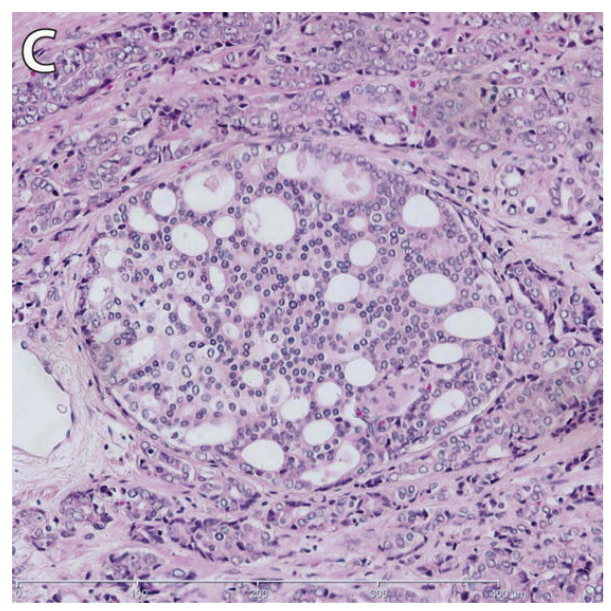

(c)

FIGURE 1: (a) intraductal carcinoma of the prostate (100x magnification). (b) 34BE12 immunohistochemistry, demonstrating the presence of basal cells supportive for intraductal carcinoma (100x magnification). (c) Cribriform growth pattern of Gleason grade 4 adenocarcinoma (200x magnification).

pathologists were not obligated to routinely report on presence of perineural invasion; there were striking differences in the frequency of perineural invasion when biopsies were reviewed for study purpose. Interestingly, only 43\% (18/42) of surveyed urologists think that presence of perineural invasion on prostate biopsy should influence treatment [48]. Ten out of 18 surveyed urologists (56\%) indicated that it helps planning nerve-sparing surgery. In contrast, nervesparing surgery was considered as a confounding factor in the studies mentioned by Harnden et al. [47]. However, despite limitations Harden et al. conclude that the weight of evidence in studies ascribing prognostic significance to perineural invasion appears to suggest that perineural invasion should influence clinical decision-making [47]. For instance, Quinn et al. demonstrated in a large cohort $(n=696)$ that perineural invasion was a significant predictor for outcome in a multivariable analysis [49]. Delancey et al. demonstrate that perineural invasion on prostatic needle-biopsy is an independent predictor for biochemical recurrence, disease-specific survival, and overall survival after radical prostatectomy [50].
2.4. Intraductal Carcinoma: A High-Risk Lesion. Intraductal carcinoma of the prostate is defined as a well-circumscribed lesion surrounded by an intact basal cell layer distended by overtly malignant-appearing epithelial populations [51] (Figures 1(a) and 1(b)). Intraductal carcinoma forms a morphologic continuum with high-grade prostate intraepithelial neoplasia (PIN), which is the generally accepted precursor of prostate cancer. While PIN is recognized by the presence of cytologically malignant cells in preexistent prostate glands of normal architecture, these glands should be enlarged in intraductal carcinoma. In contrast to PIN, which can be an isolated finding on prostate biopsy, intraductal carcinoma is associated in $90 \%$ of cases with invasive carcinoma, mostly of intermediate or high risk [52]. Up to date, three studies on intraductal carcinoma all demonstrated its independent predictive value for biochemical recurrence after radical prostatectomy, treatment failure, and distant metastasis in biopsies [53-55]. van der Kwast et al. showed that intraductal carcinoma on needle-biopsies prior to external beam radiotherapy with or without androgen deprivation therapy 
strongly predicted biochemical recurrence and early distant metastasis in a cohort of 118 intermediate and 132 high risk prostate cancer patients [55].

2.5. Percentage Gleason Grades 4 and 5. A proposed adaption to the Gleason grading system by Vis et al. is the reporting of percentage of Gleason grades $4 / 5$ in prostatic needlebiopsies [37]. In this study, containing 281 patients, amount of high-grade cancer (length in $\mathrm{mm}$, or percentage) in the core biopsy was an independent prognostic factor for biochemical recurrence and clinical relapse of prostate cancer [37]. In addition, when biopsy Gleason score 7 cancers were subcategorized into $3+4$ and $4+3$ cancers, the amount of high-grade cancer was the strongest predictor of biochemical recurrence free survival, whereas the Gleason grading system was rejected in the presence of high-grade components [37]. So far, no other studies have yet validated the predictive value of this parameter on prostatic needle-biopsies.

\subsection{Gleason Grade 4 Patterns: Individual Prognostic Value.} Gleason grade 4 tumors represent a diverse group, containing at least 4 distinctive growth patterns: fused, cribriform, illdefined, and glomeruloid $[1,14,56]$. Recently, Dong et al. studied the prognostic value of these distinct Gleason grade 4 patterns and showed that cribriform growth, in particular, was strongly associated with biochemical recurrence and distant metastasis after radical prostatectomy [57] (Figure 1(c)). Only one publication has yet demonstrated the adverse prognostic value of cribriform growth pattern in a biopsy series, with radiotherapy as treatment, and biochemical recurrence as clinical endpoint [54].

2.7. Extraprostatic Extension. Rarely, prostate cancer is infiltrating extraprostatic fat tissue, seminal vesicle, or ejaculatory ducts on needle-biopsies. Fat invasion by tumor on prostatic needle-biopsy is considered as extraprostatic extension by $81 \%$ of surveyed pathologists from the European Network of Uropathology [58]. If present, these features should be mentioned in diagnostic needle-biopsies. Since signs of extraprostatic extension are mostly seen in voluminous prostate cancer with high Gleason score, these patients could be less eligible for radical treatment.

2.8. Tumor-Associated Macrophages. Solid tumors grow in a complex and dynamic stroma involving various cell types, for example, leukocytes, fibroblasts, and endothelial cells. Tumor-associated macrophages are part of the tumor microenvironment and seem to influence solid tumor progression, for example, in colon, breast, and ovarian cancer [59]. Nonomura et al. were the first to study its prognostic relevance in prostate biopsies [60]. The presence of tumorassociated macrophages, as immunohistochemically identified by the CD68 antibody in a cohort of 71 prostate cancer patients treated with hormones, was associated with disease recurrence after hormonal therapy. Furthermore, tumorassociated macrophages count ( $>22$ at $400 \mathrm{x}$ magnification), PSA level, Gleason score $\geq 7$, and extracapsular extension were independent predictors for biochemical recurrence free survival in multivariate analysis [60]. Studies on tumorinfiltrating lymphocytes in prostatic needle-biopsies have not yet been performed.

\subsection{Summary}

(i) To date, Gleason grading on prostatic biopsy is the most important predictor for biochemical recurrence, distant metastasis, and cancer-specific mortality in prostate cancer.

(ii) Despite the modification in 2005 by the ISUP, Gleason score upgrading at radical prostatectomy still ranges between 26 and $50 \%$.

(iii) Proposed predictors of upgrading are high preoperative PSA, larger tumor percentage per biopsy core, number of positive cores, and presence of perineural invasion.

(iv) Proposed predictors of downgrading are smaller tumor percentage per core and large prostate volume.

(v) Differing Gleason scores on prostate needle biopsies could be a useful tool in decreasing the odds of upgrading.

(vi) Tumor quantification is an important prognostic factor and implemented in clinical nomograms of prostate cancer; there is, however, no consensus on the best methodology for its assessment. Furthermore, despite its fundamental role in clinical nomograms, the effect on survival is relatively small.

(vii) Presence of perineural invasion on needle-biopsies is an independent predictor for survival, and therefore a recommended parameter to add in standardized pathology reporting.

(viii) The amount of Gleason grade 4/5 in needle-biopsies seems to have prognostic value; however, it needs to be validated in large cohorts with strong endpoints.

(ix) Cribriform growth and/or intraductal spread are promising histopathological markers in needle-biopsies.

(x) The prognostic value of tumor-associated inflammation has recently been acknowledged in multiple solid tumors and needs to be further investigated in prostate cancer.

\section{Molecular Markers}

3.1. Ki-67. The Ki-67 protein is a cell proliferation marker, which is expressed in $G_{1}, S, G_{2}$, and $M$ phases of the cell cycle being absent in resting $\left(\mathrm{G}_{0}\right)$ cells. The Ki-67 labeling index as determined by the MIB-1 antibody is the best studied prostate cancer marker in needle-biopsies up to date [6166]. Ki-67 labeling index shows a strong correlation with Gleason score on diagnostic biopsies $[61,63]$, on subsequent radical prostatectomy [64-66], or both [62]. In a cohort of 91 patients, Rubio et al. found Ki-67 (cut-off value of 5\% positive nuclei) on needle-biopsies to be a marker for diseasefree survival after radical prostatectomy in univariate analysis 
[62]. Zellweger and colleagues found that Ki-67 labeling index of $\geq 10 \%$ at biopsy cores in 279 patients independently predicted seminal vesicle invasion and Gleason score on subsequent radical prostatectomy [66]. In addition, they found that Ki-67 was the only independent marker for postoperative biochemical recurrence in a subgroup of lowvolume $(<7 \%)$ or low-grade (Gleason score $<7$ ) prostate cancer at needle-biopsies. Tolonen et al. demonstrated that Ki-67 in 247 primarily endocrine-treated prostate cancer patients was associated with progression-free survival [63]. Ki-67 labeling $\geq 10 \%$ on 293 microarrayed needle-biopsies had independent predictive value for disease-specific death together with Gleason score and PSA [61]. Recently, Tollefson et al. calculated in a cohort of 451 prostate cancer needlebiopsies that every $1 \%$ increase in Ki-67 expression resulted in a $12 \%$ increased risk of cancer-specific death after radical prostatectomy [29]. Whereas these studies all show additional value of Ki-67 expression in needle-biopsies with aggressive disease features, two studies focused on the relation of Ki-67 labeling in needle-biopsies and presence of indolent disease on radical prostatectomy. In a well-defined screening cohort Vis et al. were not able to find a significant association of Ki$67 \geq 10 \%$ with significant prostate cancer at radical prostatectomy in 81 patients [64]. Wolters et al. were also not able to find a significant association of high Ki-67 expression (>3\%) with significant prostate cancer at radical prostatectomy in 86 patients [65]. Taken together, enhanced Ki-67 labeling at needle-biopsy is associated with adverse clinicopathologic features and disease-specific death in general prostate cancer populations.

3.2. $p 27 . \mathrm{p} 27^{\mathrm{kip} 1}$ is a cyclin-dependent kinase (cdk) inhibitor. It inhibits cell cycle progression in $G_{1}$ phase by preventing activation of cyclin E-cdk2 and cyclin D/-cdk4 complexes [67]. Loss of p27 has been widely associated with progression of different tumor types, including breast, colorectal, and lung cancer [67]. Generally, p27 expression in prostate cancer needle-biopsies correlates well with the p27 labeling in radical prostatectomy samples [64, 67]. In prostate cancer, various groups have shown that loss of p27 expression was associated with more aggressive disease parameters. Thomas et al. demonstrated that low expression $(<30 \%)$ of p27 in needlebiopsies correlated with higher Gleason score and pT-stage at radical prostatectomy [67]. In this relatively small cohort of 44 patients, tumors with low p27 expression showed a trend towards shortened biochemical recurrence free survival after operation. Vis et al. showed that p27 expression in $<50 \%$ together with Gleason score were the only significant parameters to predict clinically significant disease at radical prostatectomy in a screen-detected cohort of 81 prostate cancer patients [64]. In addition, we found that p27 in $<90 \%$ in a low-risk prostate cancer cohort was an independent parameter of clinically significant prostate cancer in 86 radical prostatectomy samples [65]. Therefore, loss of p27 is a marker of more aggressive prostate cancer, although the number of patients is limited and different standard cutoff levels have been used by various groups.
3.3. EZH2. Enhancer of zeste homologue 2 (EZH2) belongs to the Polycomb-group proteins and is important in maintaining cell identity and regulation of the cell cycle [68, 69]. EZH2 has been reported to be of both prognostic and therapeutic value in different tumors, such as small cell lung carcinoma [70], breast cancer [71, 72], cervical carcinomas [73], urinary tract carcinoma [74], and lymphoma [75]. Through gene expression profiling, EZH2 was found to be overexpressed in hormone-refractory metastatic prostate cancer [69]. Overexpression of EZH2 in radical prostatectomy samples was associated with poor prognosis $[69,76-$ $78]$. In a set of 86 needle-biopsies of screen-detected lowrisk prostate cancer, EZH2 expression $>1 \%$ was associated with clinically significant tumors on radical prostatectomy, defined as presence of extraprostatic extension, Gleason grade $4 / 5$ or tumor volume $\geq 0.5 \mathrm{~mL}$ [65]. In the same study, no prognostic value was found for Polycomb-group protein BMI1. Tolonen et al. found independent predictive value for EZH2 (expression level of $>15 \%$ ) for progressionfree survival in 247 hormone-treated biopsies [63]. While enhanced EZH2 expression in prostate cancer biopsies has independent prognostic value, there is no consensus yet on cutoff points in clinical practice.

3.4. TMPRSS2:ERG. Fusion of the androgen-dependent TMPRSS2 gene to ETS-transcription factor ERG (TMPRSS2 $: E R G)$ is one of the most common genetic alterations in prostate cancer occurring in 50\%-70\% of tumors [79]. Many groups have analyzed the presence of TMPRSS2:ERG fusion or ERG protein expression in prostate cancer cohorts with variable outcome [80-92]. Barros-Silva et al. used fluorescent in situ hybridization (FISH) to detect TMPRSS2-ERG rearrangement in a cohort of 200 biopsies and found an association with low PSA levels at diagnosis and low Gleason score [93]. In needle-biopsies immunohistochemical ERG detection can be used to discriminate prostate cancer from its mimickers, although the additional value to other markers such as p63, basal cell keratin 5, and AMACR is limited [92, 94-99]. In an active surveillance cohort of 265 men, Berg et al. found a strong correlation between ERG protein expression and disease-progression [100]. Likewise, Hagglof et al. showed a shorter survival of prostate cancer patients on watchful waiting when ERG was expressed [101]. They found a cumulative 2-year progression rate of $59 \%$ in the ERG-positive group versus $22 \%$ in the ERG-negative group. Finally, expression of ERG in high-grade PIN was associated with a higher chance of developing prostate cancer at subsequent biopsies [102]. ERG immunohistochemistry is an easy to perform methodology for detecting TMPRSS2:ERG fusion in prostate cancer. While the clinical relevance of TMPRSS2:ERG fusion on radical prostatectomy specimens is unresolved yet, most reports indicate that ERG expression on biopsy, in surveillance cohorts can select a subgroup with higher chance to progression.

3.5. Neuroendocrine Differentiation. In many prostate cancers, scattered tumor cells show neuroendocrine differentiation as demonstrated by antibodies to Chromogranine, 
Synaptophysine, or Serotonine. After hormonal therapy, the relative number of neuroendocrine cells is increased, putatively due to their androgen-independent nature [103-105]. Despite extensive studies on neuroendocrine differentiation in relation to castration-resistance, this feature is rarely studied in pretreatment biopsies. Krauss and colleagues have shown that Chromogranine An expression of $>1 \%$ in prostate cancer biopsies is an independent predictor for distant metastasis and cause-specific survival after primary radiation therapy $[106,107]$.

3.6. $c-M Y C$. The oncogene $c-M Y C$ located at $8 \mathrm{q} 24$ encodes a transcription factor involved in cell cycle progression, cell growth, proliferation, protein synthesis, mitochondrial function, stem cell renewal, and DNA replication [108, 109]. c-MYC is amplified in approximately $70 \%$ of clinical prostate cancer $[93,110,111]$. Ribeiro et al. found that patients with gain of MYC gene copy numbers in a group of 60 prostate cancer needle-biopsies using FISH were significantly at risk for disease-specific death [110]. Bastacky et al. showed that amplification of $c-M Y C$ in needle-biopsies with high-grade PIN was predictive of finding prostate cancer in subsequent biopsies [112]. The potential predictive value of $c-M Y C$ was confirmed by Zafarana et al. in a cohort of 126 needle biopsies, where they found $c-M Y C$ gain alone to be prognostic for tumor recurrence after radiotherapy [111]. $c-M Y C$ gain combined with loss of PTEN further increased the predictive value for recurrence after radiotherapy.

3.7. PTEN. Phosphatase and tensin homologue (PTEN) is a tumor suppressor gene which is inactivated in many different tumors, including prostate cancer [113]. On large cohorts of radical prostatectomy samples and transurethral resection (TUR) samples, PTEN loss has been associated with bone metastases, resistance to radiotherapy and chemotherapy, progression to androgen-independent disease, and disease recurrence after surgery $[113,114]$. Zafarana et al. found that $P T E N$ loss alone and in combination with $c$-MYC gain were independently associated with biochemical recurrence after radiation therapy in a group of 126 intermediate-risk prostate cancer biopsies [111].

3.8. APC. Using quantitative methylation-specific PCR (QMSP) Henrique and colleagues showed that hypermethylation of APC, GSTP1, and RASSF1A in 83 prostate cancers at sextant needle-biopsies was associated with poor diseasespecific survival [115]. Besides clinical stage, hypermethylation of $A P C$ was independently predictive for decreased disease-free and disease-specific survival. Methylation of CCND2 and RARB2 in the same study did not have predictive value for disease outcome.

3.9. Molecular Signatures. Subgroups with unique molecular, pathologic, clinical, and therapy-sensitivity, as have been defined in breast cancer, have not been delineated in prostate cancer yet. Nevertheless, recently gene signatures have been put forth to predict prostate cancer behavior. Klein et al. demonstrated that a 17-gene assay was able to identify patients with high-grade and high-stage disease at radical prostatectomy in a cohort of 395 men with low- to intermediaterisk prostate cancer at biopsies [116]. In addition, Irshad et al. identified a 3-gene signature of FGFR1, PMP22, and $C D K N 1 A$, which could accurately predict the outcome of low Gleason score prostate cancer in different cohorts [117]. For clinical implementation and validation this group applied immunohistochemistry for the respective proteins. In a limited matched cohort of 43 low-risk prostate cancer patients on active surveillance they were perfectly able to identify patients with failure upon active surveillance by reduced expression of these 3 proteins. Gene-based signatures therefore are a promising tool for risk stratification and might gain wider application if translation to easy-to-use procedures such as immunohistochemistry is available.

3.10. Biopsy Markers without Prognostic Value. While numerous markers have been shown to correlate with adverse clinicopathologic parameters on radical prostatectomy, just a limited number of these has been investigated in pretreatment needle-biopsies. Briefly, we also want to mention markers that have been investigated on biopsies but did not show additional value. In a group of 91 prostate cancer needlebiopsies, Bax, Bcl-2, and Cox-2 did not show independent predictive value for disease-free survival, although Cox-2 was predictive in univariate analysis [62]. On a large cohort of 247 patients with primary endocrine treatment, Tolonen et al. demonstrated that minichromosome maintenance protein 7 (MCM7) was a significant albeit not independent marker for disease-progression [63]. We validated the prognostic value of Cystein-rich secretory protein 3 (CRISP-3) and $\beta$-Microseminoprotein $(\beta$-MSP) in a screening cohort of 174 men. We found that expression of these markers was correlated with Gleason score, tumor volume, and pT-stage and significant disease on subsequent radical prostatectomy samples but were not able to predict recurrence [118].

\subsection{Summary}

(i) The cell proliferation marker Ki-67 (MIB-1) is the best studied immunohistochemical marker in prostate with independent prognostic value in multiple studies.

(ii) Cyclin-dependent kinase inhibitor p27 and Polycomb-group protein EZH2 are both promising immunohistochemical markers for predicting disease outcome.

(iii) The clinical significance of TMPRSS:ERG fusion or ERG protein overexpression is still controversial, although some studies demonstrate adverse prognostic value in active surveillance/watchful waiting cohorts.

(iv) Amplification of $c$-MYC, PTEN loss, and APC hypermethylation are promising markers for predicting disease-specific death, albeit only demonstrated in a small number of biopsy cohorts. 
(v) Identification of complex gene signatures offers novel promising platforms for predicting disease-outcome. Routine implementation in local pathology laboratories is currently not applicable.

\section{Conclusions}

Investigation of potential novel predictive markers in prostate cancer needle-biopsies is of importance to affect clinical decision-making and to be implemented in daily practice. A prerequisite in analyzing novel markers on needle-biopsies is the presence of well-characterized patient cohorts with clinical follow-up and availability of prostate cancer tissue for actual testing. Secondary and tertiary cancer centers often do not have original tissue blocks for further research on site. In addition, small foci of prostate cancer are often not present anymore in the remaining paraffin block. Incorporation of both detailed histopathological prostate cancer features and molecular markers can support optimal therapeutic decisionmaking in individual patients.

Comprehensive reporting of novel histopathological parameters such as percentage Gleason grade 4/5, intraductal carcinoma and potentially Gleason grade 4 growth pattern is a fast and cheap way to better estimate a prostate cancer's future clinical behavior in daily practice. Various molecular markers such as Ki-67, p27, EZH2, and ERG immunohistochemistry, as well as $c-M Y C$ and PTEN in situ hybridization, can putatively sustain and improve pathologic diagnosis. The variability in patient cohorts, clinical endpoints, technical methodology, and quantification, however, require prospective studies in well-characterized patient groups before implementation in daily practice is feasible. Identification of complex gene-signatures is a recent and promising tool in stratification of prostate cancer patients, though still costly and not easily applicable in daily practice.

Last decade, the diagnosis of prostate cancer has changed significantly. Pathologic Gleason scoring has been modified; sextant biopsies have widely been replaced by 10 to 12 or more biopsy sampling protocols; image-guided biopsies supplement established biopsy schemes and facilitate sampling of biologically relevant tumor areas. It is difficult to interpret the additional value of established prognostic factors of earlier studies in contemporary prostate cancer health care. We advocate that incorporation of novel histopathological parameters such as percentage Gleason grade 4/5, presence of intraductal carcinoma, and Gleason grade 4 growth patterns in daily pathology practice as well as prostate cancer studies offer an inexpensive and short-term opportunity to improve prostate cancer health care and interpret the additional value of promising molecular markers.

\section{Disclosure}

A. Marije Hoogland and Charlotte F. Kweldam shared first author.

\section{Conflict of Interests}

The authors declare that there is no conflict of interests regarding the publication of this paper.

\section{References}

[1] J. I. Epstein, W. C. Allsbrook Jr., M. B. Amin et al., “The 2005 International Society of Urological Pathology (ISUP) consensus conference on Gleason grading of prostatic carcinoma," The American Journal of Surgical Pathology, vol. 29, no. 9, pp. 12281242, 2005.

[2] T. van der Kwast, L. Bubendorf, C. Mazerolles et al., "Guidelines on processing and reporting of prostate biopsies: the 2013 update of the pathology committee of the European randomized study of screening for prostate cancer (ERSPC)," Virchows Archiv, vol. 463, no. 3, pp. 367-377, 2013.

[3] M. R. Cooperberg, J. M. Broering, and P. R. Carroll, “Time trends and local variation in primary treatment of localized prostate cancer," Journal of Clinical Oncology, vol. 28, no. 7, pp. 1117-1123, 2010.

[4] M. R. Cooperberg, P. R. Carroll, and L. Klotz, "Active surveillance for prostate cancer: progress and promise," Journal of Clinical Oncology, vol. 29, no. 27, pp. 3669-3676, 2011.

[5] M. S. Soloway, C. T. Soloway, A. Eldefrawy, K. Acosta, B. Kava, and M. Manoharan, "Careful selection and close monitoring of low-risk prostate cancer patients on active surveillance minimizes the need for treatment," European Urology, vol. 58, no. 6, pp. 831-835, 2010.

[6] J. J. Tosoian, B. J. Trock, P. Landis et al., "Active surveillance program for prostate cancer: an update of the Johns Hopkins experience," Journal of Clinical Oncology, vol. 29, no. 16, pp. 2185-2190, 2011.

[7] N. J. van As, A. R. Norman, K. Thomas et al., "Predicting the probability of deferred radical treatment for localised prostate cancer managed by active surveillance," European Urology, vol. 54, no. 6, pp. 1297-1305, 2008.

[8] R. C. N. van den Bergh, S. Roemeling, M. J. Roobol et al., "Outcomes of men with screen-detected prostate cancer eligible for active surveillance who were managed expectantly," European Urology, vol. 55, no. 1, pp. 1-8, 2009.

[9] L. Egevad, A. S. Ahmad, F. Algaba et al., "Standardization of Gleason grading among 337 European pathologists," Histopathology, vol. 62, no. 2, pp. 247-256, 2013.

[10] J. K. McKenney, J. Simko, M. Bonham et al., “The potential impact of reproducibility of gleason grading in men with early stage prostate cancer managed by active surveillance: a multiinstitutional study," Journal of Urology, vol. 186, no. 2, pp. 465469, 2011.

[11] D. F. Gleason, "Classification of prostatic carcinomas.," Cancer Chemotherapy Reports, vol. 50, no. 3, pp. 125-128, 1966.

[12] J. I. Epstein, "An update of the Gleason grading system," Journal of Urology, vol. 183, no. 2, pp. 433-440, 2010.

[13] M. Latour, M. B. Amin, A. Billis et al., "Grading of invasive cribriform carcinoma on prostate needle biopsy: an interobserver study among experts in genitourinary pathology," The American Journal of Surgical Pathology, vol. 32, no. 10, pp. 1532-1539, 2008.

[14] T. L. Lotan and J. I. Epstein, "Gleason grading of prostatic adenocarcinoma with glomeruloid features on needle biopsy," Human Pathology, vol. 40, no. 4, pp. 471-477, 2009.

[15] A. Billis, M. S. Guimaraes, L. L. L. Freitas, L. Meirelles, L. A. Magna, and U. Ferreira, "The impact of the 2005 international society of urological pathology consensus conference on standard Gleason grading of prostatic carcinoma in needle biopsies," Journal of Urology, vol. 180, no. 2, pp. 548-553, 2008. 
[16] B. Helpap and L. Egevad, "The significance of modified Gleason grading of prostatic carcinoma in biopsy and radical prostatectomy specimens," Virchows Archiv, vol. 449, no. 6, pp. 622-627, 2006.

[17] P. Zareba, J. Zhang, A. Yilmaz, and K. Trpkov, "The impact of the 2005 International Society of Urological Pathology (ISUP) consensus on Gleason grading in contemporary practice," Histopathology, vol. 55, no. 4, pp. 384-391, 2009.

[18] H. Uemura, K. Hoshino, T. Sasaki et al., "Usefulness of the 2005 International Society of Urologic Pathology Gleason grading system in prostate biopsy and radical prostatectomy specimens," BJU International, vol. 103, no. 9, pp. 1190-1194, 2009.

[19] J. I. Epstein, Z. Feng, B. J. Trock, and P. M. Pierorazio, "Upgrading and downgrading of prostate cancer from biopsy to radical prostatectomy: incidence and predictive factors using the modified gleason grading system and factoring in tertiary grades," European Urology, vol. 61, no. 5, pp. 1019-1024, 2012.

[20] A. S. Moussa, M. W. Kattan, R. Berglund, C. Yu, K. Fareed, and J. S. Jones, "A nomogram for predicting upgrading in patients with low-and intermediate-grade prostate cancer in the era of extended prostate sampling," BJU International, vol. 105, no. 3 , pp. 352-358, 2010.

[21] A. S. Moussa, J. Li, M. Soriano, E. A. Klein, F. Dong, and J. S. Jones, "Prostate biopsy clinical and pathological variables that predict significant grading changes in patients with intermediate and high grade prostate cancer," BJU International, vol. 103, no. 1, pp. 43-48, 2009.

[22] H. Visapää, K. Hotakainen, J. Lundin, M. Ala-Opas, and U. Stenman, "The proportion of free PSA and upgrading of biopsy gleason score after radical prostatectomy," Urologia Internationalis, vol. 84, no. 4, pp. 378-381, 2010.

[23] J. G. Phillips, A. A. Aizer, M. H. Chen et al., "The effect of differing gleason scores at biopsy on the odds of upgrading and the risk of death from prostate cancer," Clinical Genitourinary Cancer, 2014.

[24] J. M. Whitson, S. P. Porten, J. E. Cowan, J. P. Simko, M. R. Cooperberg, and P. R. Carroll, "Factors associated with downgrading in patients with high grade prostate cancer," Urologic Oncology: Seminars and Original Investigations, vol. 31, no. 4, pp. 442-447, 2013.

[25] P. M. Pierorazio, A. E. Ross, B. M. Lin et al., "Preoperative characteristics of high-Gleason disease predictive of favourable pathological and clinical outcomes at radical prostatectomy," BJU International, vol. 110, no. 8, pp. 1122-1128, 2012.

[26] J. Busch, K. Hamborg, H. Meyer et al., "Value of prostate specific antigen density and percent free prostate specific antigen for prostate cancer prognosis," Journal of Urology, vol. 188, no. 6, pp. 2165-2170, 2012.

[27] A. Forgues, F. Rozet, F. Audenet et al., "Oncologic outcomes after minimally invasive radical prostatectomy in patients with seminal vesicle invasion (pT3b) without adjuvant therapy," World Journal of Urology, vol. 32, no. 2, pp. 519-524, 2014.

[28] J. J. Meeks, A. C. Maschino, K. T. McVary, and J. S. Sandhu, "Clinically significant prostate cancer is rarely missed by ablative procedures of the prostate in men with prostate specific antigen less than $4 \mathrm{ng} / \mathrm{ml}$," Journal of Urology, vol. 189, no. 1, pp. 111-115, 2013.

[29] M. K. Tollefson, R. J. Karnes, E. D. Kwon et al., "Prostate cancer Ki-67 (MIB-1) expression, perineural invasion, and gleason score as biopsy-based predictors of prostate cancer mortality: the Mayo model," Mayo Clinic Proceedings, vol. 89, pp. 308-318, 2014.
[30] E. W. Steyerberg, M. J. Roobol, M. W. Kattan, T. H. van der Kwast, H. J. de Koning, and F. H. Schröder, "Prediction of indolent prostate cancer: validation and updating of a prognostic nomogram," Journal of Urology, vol. 177, no. 1, pp. 107-112, 2007.

[31] R. C. van den Bergh, H. U. Ahmed, C. H. Bangma, M. R. Cooperberg, A. Villers, and C. C. Parker, "Novel tools to improve patient selection and monitoring on active surveillance for lowrisk prostate cancer: a systematic review," European Urology, vol. 65, pp. 1023-1031, 2014.

[32] M. A. Dall'Era, P. C. Albertsen, C. Bangma et al., "Active surveillance for prostate cancer: a systematic review of the literature," European Urology, vol. 62, no. 6, pp. 976-983, 2012.

[33] S. J. Freedland, W. J. Aronson, M. K. Terris et al., "The percentage of prostate needle biopsy cores with carcinoma from the more involved side of the biopsy as a predictor of prostate specific antigen recurrence after radical prostatectomy: results from the Shared Equal Access Regional Cancer Hospital (SEARCH) database," Cancer, vol. 98, no. 11, pp. 2344-2350, 2003.

[34] S. J. Freedland, W. J. Aronson, M. K. Terris et al., "Percent of prostate needle biopsy cores with cancer is a significant independent predictor of prostate specific antigen recurrence following radical prostatectomy: results from the SEARCH database," Journal of Urology, vol. 169, no. 6, pp. 2136-2141, 2003.

[35] P. W. Linson, A. K. Lee, T. Doytchinova et al., "Percentage of core lengths involved with prostate cancer: does it add to the percentage of positive prostate biopsies in predicting postoperative prostate-specific antigen outcome for men with intermediate-risk prostate cancer?" Urology, vol. 59, no. 5, pp. 704-708, 2002.

[36] C. P. Nelson, R. L. Dunn, J. T. Wei, M. A. Rubin, J. E. Montie, and M. G. Sanda, "Contemporary preoperative parameters predict cancer-free survival after radical prostatectomy: a tool to facilitate treatment decisions," Urologic Oncology, vol. 21, no. 3, pp. 213-218, 2003.

[37] A. N. Vis, S. Roemeling, R. Kranse, F. H. Schröder, and T. H. van der Kwast, "Should we replace the Gleason score with the amount of high-grade prostate cancer?," European Urology, vol. 51, no. 4, pp. 931-939, 2007.

[38] S. M. Vance, M. H. Stenmark, K. Blas, S. Halverson, D. A. Hamstra, and F. Y. Feng, "Percentage of cancer volume in biopsy cores is prognostic for prostate cancer death and overall survival in patients treated with dose-escalated external beam radiotherapy," International Journal of Radiation Oncology Biology Physics, vol. 83, no. 3, pp. 940-946, 2012.

[39] S. G. Williams, M. K. Buyyounouski, T. Pickles et al., "Percentage of biopsy cores positive for malignancy and biochemical failure following prostate cancer radiotherapy in 3,264 men: statistical significance without predictive performance," International Journal of Radiation Oncology Biology Physics, vol. 70, no. 4, pp. 1169-1175, 2008.

[40] S. J. Freedland, M. K. Terris, G. S. Csathy et al., "Preoperative model for predicting prostate specific antigen recurrence after radical prostatectomy using percent of biopsy tissue with cancer, biopsy Gleason grade and serum prostate specific antigen," The Journal of Urology, vol. 171, no. 6 I, pp. 2215-2220, 2004.

[41] H. Huland, P. Hammerer, R. Henke, and E. Huland, "Preoperative prediction of tumor heterogeneity and recurrence after radical prostatectomy for localized prostatic carcinoma with digital rectal examination, prostate specific antigen and the results of 6 systematic biopsies," Journal of Urology, vol. 155, no. 4, pp. 1344-1347, 1996. 
[42] V. Ravery, C. Chastang, M. Toublanc, L. Boccon-Gibod, and V. Delmas, "Percentage of cancer on biopsy cores accurately predicts extracapsular extension and biochemical relapse after radical prostatectomy for T1-T2 prostate cancer," European Urology, vol. 37, no. 4, pp. 449-455, 2000.

[43] J. Huang, F. A. Vicini, S. G. Williams et al., "Percentage of positive biopsy cores: a better risk stratification model for prostate cancer?" International Journal of Radiation Oncology, Biology, Physics, vol. 83, no. 4, pp. 1141-1148, 2012.

[44] S. Karram, B. J. Trock, G. J. Netto, and J. I. Epstein, "Should intervening benign tissue be included in the measurement of discontinuous foci of cancer on prostate needle biopsy? Correlation with radical prostatectomy findings," The American Journal of Surgical Pathology, vol. 35, no. 9, pp. 1351-1355, 2011.

[45] L. Schultz, C. E. Maluf, R. C. da Silva, H. Falashi Rde, M. V. da Costa, and M. I. Schultz, "Discontinuous foci of cancer in a single core of prostatic biopsy: when it occurs and performance of quantification methods in a private-practice setting," American Journal of Surgical Pathology, vol. 37, pp. 18311836, 2013.

[46] T. H. van der Kwast and M. J. Roobol, "Defining the threshold for significant versus insignificant prostate cancer," Nature Reviews Urology, vol. 10, no. 8, pp. 473-482, 2013.

[47] P. Harnden, M. D. Shelley, H. Clements et al., "The prognostic significance of perineural invasion in prostatic cancer biopsies: a systematic review," Cancer, vol. 109, no. 1, pp. 13-24, 2007.

[48] M. A. Rubin, T. A. Bismar, S. Curtis, and J. E. Montie, "Prostate needle biopsy reporting: how are the surgical members of the society of urologic oncology using pathology reports to guide treatment of prostate cancer patients?" American Journal of Surgical Pathology, vol. 28, no. 7, pp. 946-952, 2004.

[49] D. I. Quinn, S. M. Henshall, P. C. Brenner et al., "Prognostic significance of preoperative factors in localized prostatecarcinoma treated with radical prostatectomy: importance of percentage of biopsies that contain tumor and the presence of biopsy perineural invasion," Cancer, vol. 97, no. 8, pp. 1884-1893, 2003.

[50] J. O. Delancey, D. P. Wood Jr., C. He et al., "Evidence of perineural invasion on prostate biopsy specimen and survival after radical prostatectomy," Urology, vol. 81, no. 2, pp. 354-357, 2013.

[51] M. Pickup and T. H. Van Der Kwast, "My approach to intraductal lesions of the prostate gland," Journal of Clinical Pathology, vol. 60, no. 8, pp. 856-865, 2007.

[52] B. D. Robinson and J. I. Epstein, "Intraductal carcinoma of the prostate without invasive carcinoma on needle biopsy: Emphasis on radical prostatectomy findings," Journal of Urology, vol. 184, no. 4, pp. 1328-1333, 2010.

[53] R. J. Cohen, W. C. Chan, S. G. Edgar et al., "Prediction of pathological stage and clinical outcome in prostate cancer: an improved pre-operative model incorporating biopsy-determined intraductal carcinoma," British Journal of Urology, vol. 81, no. 3, pp. 413-418, 1998.

[54] E. Efstathiou, N. A. Abrahams, R. F. Tibbs et al., "Morphologic characterization of preoperatively treated prostate cancer: toward a post-therapy histologic classification," European Urology, vol. 57, no. 6, pp. 1030-1038, 2010.

[55] T. van der Kwast, N. Al Daoud, L. Collette et al., "Biopsy diagnosis of intraductal carcinoma is prognostic in intermediate and high risk prostate cancer patients treated by radiotherapy," European Journal of Cancer, vol. 48, no. 9, pp. 1318-1325, 2012.

[56] J. I. Epstein, "Prostatic ductal adenocarcinoma: a mini review," Medical Principles and Practice, vol. 19, no. 1, pp. 82-85, 2010.
[57] F. Dong, P. Yang, C. Wang et al., "Architectural heterogeneity and cribriform pattern predict adverse clinical outcome for Gleason grade 4 prostatic adenocarcinoma," American Journal of Surgical Pathology, vol. 37, pp. 1855-1861, 2013.

[58] D. M. Berney, F. Algaba, P. Camparo et al., "Variation in reporting of cancer extent and benign histology in prostate biopsies among European pathologists," Virchows Archiv, vol. 464, pp. 583-587, 2014.

[59] A. Sica, T. Schioppa, A. Mantovani, and P. Allavena, "Tumourassociated macrophages are a distinct M2 polarised population promoting tumour progression: potential targets of anti-cancer therapy," European Journal of Cancer, vol. 42, no. 6, pp. 717-727, 2006.

[60] N. Nonomura, H. Takayama, M. Nakayama et al., "Infiltration of tumour-associated macrophages in prostate biopsy specimens is predictive of disease progression after hormonal therapy for prostate cancer," BJU International, vol. 107, no. 12, pp. 19181922, 2011.

[61] G. Fisher, Z. H. Yang, S. Kudahetti et al., "Prognostic value of Ki-67 for prostate cancer death in a conservatively managed cohort," British Journal of Cancer, vol. 108, no. 2, pp. 271-277, 2013.

[62] J. Rubio, D. Ramos, J. A. López-Guerrero et al., "Immunohistochemical expression of Ki-67 antigen, Cox-2 and Bax/Bcl-2 in prostate cancer; prognostic value in biopsies and radical prostatectomy specimens," European Urology, vol. 48, no. 5, pp. 745-751, 2005.

[63] T. T. Tolonen, T. L. J. Tammela, P. M. Kujala, V. J. Tuominen, J. J. Isola, and T. Visakorpi, "Histopathological variables and biomarkers enhancer of zeste homologue 2, Ki-67 and minichromosome maintenance protein 7 as prognosticators in primarily endocrine-treated prostate cancer," BJU International, vol. 108, no. 9, pp. 1430-1438, 2011.

[64] A. N. Vis, B. W. G. van Rhijn, M. A. Noordzij, F. H. Schröder, and T. H. van der Kwast, "Value of tissue markers p27kip1, MIB-1, and CD44s for the pre-operative prediction of tumour features in screen-detected prostate cancer," The Journal of Pathology, vol. 197, no. 2, pp. 148-154, 2002.

[65] T. Wolters, K. J. Vissers, C. H. Bangma, F. H. Schröder, and G. J. L. H. Van Leenders, "The value of EZH2, p27kip1, BMI-1 and MIB-1 on biopsy specimens with low-risk prostate cancer in selecting men with significant prostate cancer at prostatectomy," BJU International, vol. 106, no. 2, pp. 280-286, 2010.

[66] T. Zellweger, S. Günther, I. Zlobec et al., "Tumour growth fraction measured by immunohistochemical staining of Ki67 is an independent prognostic factor in preoperative prostate biopsies with small-volume or low-grade prostate cancer," International Journal of Cancer, vol. 124, no. 9, pp. 2116-2123, 2009.

[67] G. V. Thomas, M. I. Schrage, L. Rosenfelt et al., "Preoperative prostate needle biopsy p27 correlates with subsequent radical prostatectomy p27, gleason grade and pathological stage," Journal of Urology, vol. 164, no. 6, pp. 1987-1991, 2000.

[68] W. R. Sellers and M. Loda, "The EZH2 polycomb transcriptional repressor-a marker or mover of metastatic prostate cancer?" Cancer Cell, vol. 2, no. 5, pp. 349-350, 2002.

[69] S. Varambally, S. M. Dhanasekaran, M. Zhou et al., "The polycomb group protein EZH2 is involved in progression of prostate cancer," Nature, vol. 419, no. 6907, pp. 624-629, 2002.

[70] C. Behrens, L. M. Solis, H. Lin et al., "EZH2 protein expression associates with the early pathogenesis, tumor progression, and 
prognosis of non-small cell lung carcinoma," Clinical Cancer Research, vol. 19, pp. 6556-6565, 2013.

[71] G. Deb, V. S. Thakur, and S. Gupta, "Multifaceted role of EZH2 in breast and prostate tumorigenesis: epigenetics and beyond," Epigenetics, vol. 8, no. 5, pp. 464-476, 2013.

[72] S. Roh, S. Y. Park, H. S. Ko, J. S. Sohn, and E. J. Cha, "EZH2 expression in invasive lobular carcinoma of the breast," World Journal of Surgical Oncology, vol. 11, p. 299, 2013.

[73] Y. Liu, T. Liu, X. Bao, M. He, L. Li, and X. Yang, "Increased EZH2 expression is associated with proliferation and progression of cervical cancer and indicates a poor prognosis," The International Journal of Gynecological Pathology, vol. 33, pp. 218-224, 2014.

[74] A. Hayashi, T. Morikawa, T. Kawai et al., "Clinicopathological and prognostic significance of EZH2 expression in upper urinary tract carcinoma," Virchows Archiv, vol. 464, pp. 463471, 2014.

[75] H. J. Lee, D. H. Shin, K. B. Kim et al., "Polycomb protein EZH2 expression in diffuse large B-cell lymphoma is associated with better prognosis in patients treated with rituximab, cyclophosphamide, doxorubicin," Leukemia and Lymphoma, 2014.

[76] A. M. Hoogland, E. I. Verhoef, M. J. Roobol et al., "Validation of stem cell markers in clinical prostate cancer: alpha6-integrin is predictive for non-aggressive disease," Prostate, vol. 74, pp. 488496, 2014.

[77] S. Laitinen, P. M. Martikainen, T. Tolonen, J. Isola, T. L. J. Tammela, and T. Visakorpi, "EZH2, Ki-67 and MCM7 are prognostic markers in prostatectomy treated patients," International Journal of Cancer, vol. 122, no. 3, pp. 595-602, 2008.

[78] G. J. L. H. van Leenders, D. Dukers, D. Hessels et al., "Polycombgroup oncogenes EZH2, BMI1, and RING1 are overexpressed in prostate cancer with adverse pathologic and clinical features," European Urology, vol. 52, no. 2, pp. 455-463, 2007.

[79] S. A. Tomlins, D. R. Rhodes, S. Perner et al., "Recurrent fusion of TMPRSS2 and ETS transcription factor genes in prostate cancer," Science, vol. 310, no. 5748, pp. 644-648, 2005.

[80] R. B. Shah, "Clinical applications of novel ERG immunohistochemistry in prostate cancer diagnosis and management," Advances in Anatomic Pathology, vol. 20, no. 2, pp. 117-124, 2013.

[81] D. Hossain and D. G. Bostwick, "Significance of the TMPRSS2 :ERG gene fusion in prostate cancer," BJU International, vol. 111, no. 5, pp. 834-835, 2013.

[82] D. Hessels and J. A. Schalken, "Recurrent gene fusions in prostate cancer: their clinical implications and uses," Current Urology Reports, vol. 14, no. 3, pp. 214-222, 2013.

[83] B. Xu, M. Chevarie-Davis, S. Chevalier et al., "The prognostic role of ERG immunopositivity in prostatic acinar adenocarcinoma: a study including 454 cases and review of the literature," Human Pathology, vol. 45, pp. 488-497, 2014.

[84] J. P. Clark and C. S. Cooper, "ETS gene fusions in prostate cancer," Nature Reviews Urology, vol. 6, no. 8, pp. 429-439, 2009.

[85] B. Furusato, S.-. Tan, D. Young et al., "ERG oncoprotein expression in prostate cancer: clonal progression of ERG-positive tumor cells and potential for ERG-based stratification," Prostate Cancer and Prostatic Diseases, vol. 13, no. 3, pp. 228-237, 2010.

[86] A. M. Hoogland, G. Jenster, W. M. van Weerden et al., "ERG immunohistochemistry is not predictive for PSA recurrence, local recurrence or overall survival after radical prostatectomy for prostate cancer," Modern Pathology, vol. 25, no. 3, pp. 471479, 2012.
[87] M. Miettinen, Z. F. Wang, A. Paetau et al., "ERG transcription factor as an immunohistochemical marker for vascular endothelial tumors and prostatic carcinoma," The American Journal of Surgical Pathology, vol. 35, no. 3, pp. 432-441, 2011.

[88] K. Park, S. A. Tomlins, K. M. Mudaliar et al., "Antibody-based detection of ERG rearrangement-positive prostate cancer," Neoplasia, vol. 12, no. 7, pp. 590-598, 2010.

[89] O. R. Saramäki, A. E. Harjula, P. M. Martikainen, R. Lvessella, T. L. J. Tammela, and T. Visakorpi, "TMPRSS2.ERG fusion identifies a subgroup of prostate cancers with a favorable prognosis," Clinical Cancer Research, vol. 14, no. 11, pp. 33953400, 2008.

[90] V. J. Scheble, G. Scharf, M. Braun et al., "ERG rearrangement in local recurrences compared to distant metastases of castrationresistant prostate cancer," Virchows Archiv, vol. 461, no. 2, pp. 157-162, 2012.

[91] S. A. Tomlins, B. Laxman, S. Varambally et al., "Role of the TMPRSS2-ERG gene fusion in prostate cancer," Neoplasia, vol. 10, no. 2, pp. 177-188, 2008.

[92] G. J. van Leenders, J. L. Boormans, C. J. Vissers et al., "Antibody EPR3864 is specific for ERG genomic fusions in prostate cancer: implications for pathological practice," Modern Pathology, vol. 24, no. 8, pp. 1128-1138, 2011.

[93] J. D. Barros-Silva, F. R. Ribeiro, Â. Rodrigues et al., "Relative $8 \mathrm{q}$ gain predicts disease-specific survival irrespective of the TMPRSS2-ERG fusion status in diagnostic biopsies of prostate cancer," Genes Chromosomes and Cancer, vol. 50, no. 8, pp. 662671, 2011.

[94] C. Andrews and P. A. Humphrey, "Utility of ERG versus AMACR expression in diagnosis of minimal adenocarcinoma of the prostate in needle biopsy tissue," The American Journal of Surgical Pathology, vol. 38, pp. 1007-1012, 2014.

[95] A. Bjartell, R. Montironi, D. M. Berney, and L. Egevad, “Tumour markers in prostate cancer II: diagnostic and prognostic cellular biomarkers," Acta Oncologica, vol. 50, 1, pp. 76-84, 2011.

[96] C. Boran, E. Kandirali, F. Yilmaz, E. Serin, and M. Akyol, "Reliability of the $34 \beta \mathrm{E} 12$, keratin 5/6, p63, bcl-2, and AMACR in the diagnosis of prostate carcinoma," Urologic Oncology: Seminars and Original Investigations, vol. 29, no. 6, pp. 614-623, 2011.

[97] P. D. Dabir, P. Ottosen, S. Høyer, and S. Hamilton-Dutoit, "Comparative analysis of three- and two-antibody cocktails to AMACR and basal cell markers for the immunohistochemical diagnosis of prostate carcinoma," Diagnostic Pathology, vol. 7, no. 1 , article 81, 2012.

[98] K. Pavlakis, K. Stravodimos, T. Kapetanakis et al., "Evaluation of routine application of P504S, 34 $\beta$ E12 and p63 immunostaining on 250 prostate needle biopsy specimens," International Urology and Nephrology, vol. 42, no. 2, pp. 325-330, 2010.

[99] R. B. Shah, L. P. Kunju, R. Shen, M. LeBlanc, M. Zhou, and M. A. Rubin, "Usefulness of basal cell cocktail $(34 \beta E 12+$ p63) in the diagnosis of atypical prostate glandular proliferations," American Journal of Clinical Pathology, vol. 122, no. 4, pp. 517523, 2004.

[100] K. D. Berg, B. Vainer, F. B. Thomsen et al., "ERG protein expression in diagnostic specimens is associated with increased risk of progression during active surveillance for prostate cancer," European Urology, 2014.

[101] C. Hagglof, P. Hammarsten, K. Stromvall et al., "TMPRSS2-ERG expression predicts prostate cancer survival and associates with stromal biomarkers," PLoS One, vol. 9, Article ID e86824, 2014. 
[102] K. Park, J. T. Dalton, R. Narayanan et al., "TMPRSS2:ERG gene fusion predicts subsequent detection of prostate cancer in patients with high-grade prostatic intraepithelial neoplasia," Journal of Clinical Oncology, vol. 32, pp. 206-211, 2014.

[103] P.-A. Abrahamsson, "Neuroendocrine cells in tumour growth of the prostate," Endocrine-Related Cancer, vol. 6, no. 4, pp. 503519, 1999.

[104] P. A. di Sant'Agnese, "Neuroendocrine differentiation in carcinoma of the prostate: diagnostic, prognostic, and therapeutic implications," Cancer, vol. 70, no. 1, pp. 254-268, 1992.

[105] P. A. Di Sant'Agnese and A. T. K. Cockett, "The prostatic endocrine-paracrine (neuroendocrine) regulatory system and neuroendocrine differentiation in prostatic carcinoma: a review and future directions in basic research," Journal of Urology, vol. 152, no. 5, pp. 1927-1931, 1994.

[106] D. J. Krauss, M. Amin, B. Stone et al., "Chromogranin A staining as a prognostic variable in newly diagnosed Gleason score 710 prostate cancer treated with definitive radiotherapy," The Prostate, vol. 74, no. 5, pp. 520-527, 2014.

[107] D. J. Krauss, S. Hayek, M. Amin et al., "Prognostic significance of neuroendocrine differentiation in patients with gleason score 8-10 prostate cancer treated with primary radiotherapy," International Journal of Radiation Oncology Biology Physics, vol. 81, no. 3, pp. el19-e125, 2011.

[108] L. M. Boxer and C. V. Dang, "Translocations involving c-myc and c-myc function," Oncogene, vol. 20, no. 40, pp. 5595-5610, 2001.

[109] B. Gurel, T. Iwata, C. M. Koh et al., "Nuclear MYC protein overexpression is an early alteration in human prostate carcinogenesis," Modern Pathology, vol. 21, no. 9, pp. 1156-1167, 2008.

[110] F. R. Ribeiro, R. Henrique, A. T. Martins, C. Jeronimo, and M. R. Teixeira, "Relative copy number gain of MYC in diagnostic needle biopsies is an independent prognostic factor for prostate cancer patients," European Urology, vol. 52, pp. 116-125, 2007.

[111] G. Zafarana, A. S. Ishkanian, C. A. Malloff et al., "Copy number alterations of c-MYC and PTEN are prognostic factors for relapse after prostate cancer radiotherapy," Cancer, vol. 118, no. 16, pp. 4053-4062, 2012.

[112] S. Bastacky, K. Cieply, C. Sherer, R. Dhir, and J. I. Epstein, "Use of interphase fluorescence in situ hybridization in prostate needle biopsy specimens with isolated high-grade prostatic intraepithelial neoplasia as a predictor of prostate adenocarcinoma on follow-up biopsy," Human Pathology, vol. 35, no. 3, pp. 281-289, 2004.

[113] C. C. Uzoh, C. M. Perks, A. Bahl, J. M. P. Holly, M. Sugiono, and R. A. Persad, "PTEN-mediated pathways and their association with treatment-resistant prostate cancer," BJU International, vol. 104, no. 4, pp. 556-561, 2009.

[114] J. Cuzick, Z. H. Yang, G. Fisher et al., "Prognostic value of PTEN loss in men with conservatively managed localised prostate cancer," British Journal of Cancer, vol. 108, no. 12, pp. 2582-2589, 2013.

[115] R. Henrique, F. R. Ribeiro, D. Fonseca et al., "High promoter methylation levels of APC predict poor prognosis in sextant biopsies from prostate cancer patients," Clinical Cancer Research, vol. 13, no. 20, pp. 6122-6129, 2007.

[116] E. A. Klein, M. R. Cooperberg, C. Magi-Galluzzi et al., "A 17-gene assay to predict prostate cancer aggressiveness in the context of Gleason grade heterogeneity, tumor multifocality, and biopsy undersampling," European Urology, vol. 66, no. 3, pp. 550-560, 2014.
[117] S. Irshad, M. Bansal, M. Castillo-Martin et al., "A molecular signature predictive of indolent prostate cancer," Science Translational Medicine, vol. 5, no. 202, p. 202ra122, 2013.

[118] A. M. Hoogland, A. Dahlman, K. J. Vissers et al., "Cysteine-rich secretory protein 3 and $\beta$-microseminoprotein on prostate cancer needle biopsies do not have predictive value for subsequent prostatectomy outcome," BJU International, vol. 108, no. 8, pp. 1356-1362, 2011. 


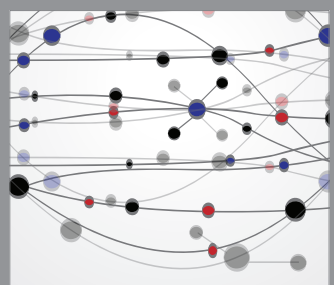

The Scientific World Journal
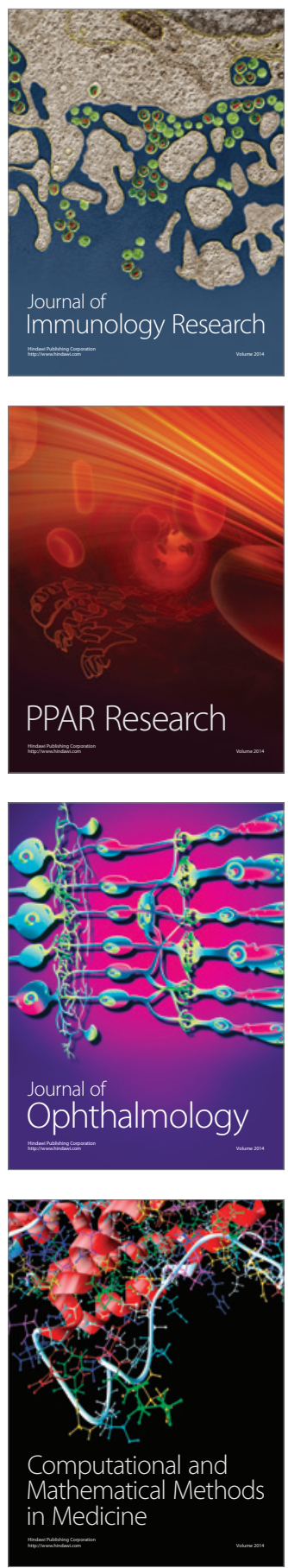

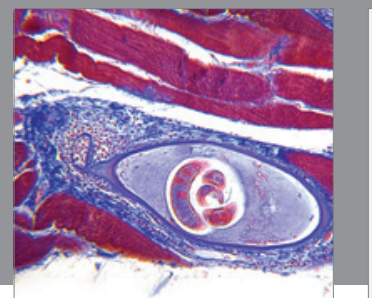

Gastroenterology

Research and Practice
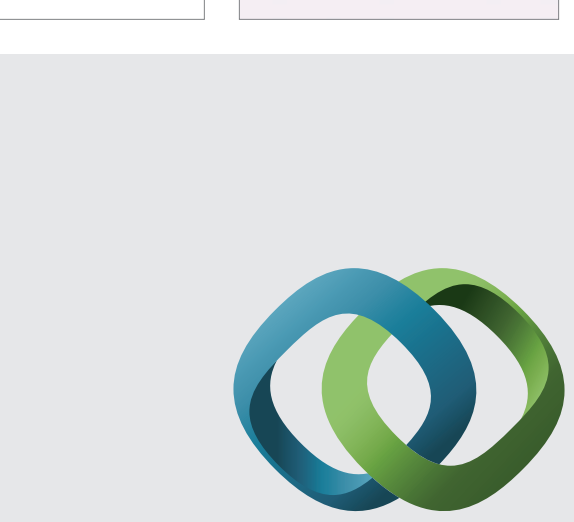

\section{Hindawi}

Submit your manuscripts at

http://www.hindawi.com
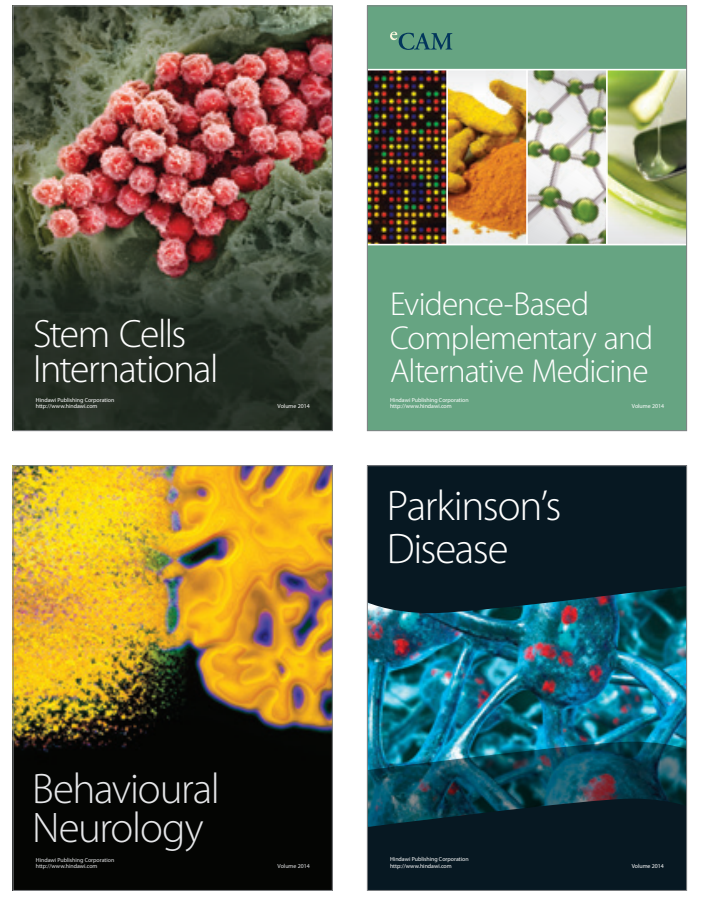
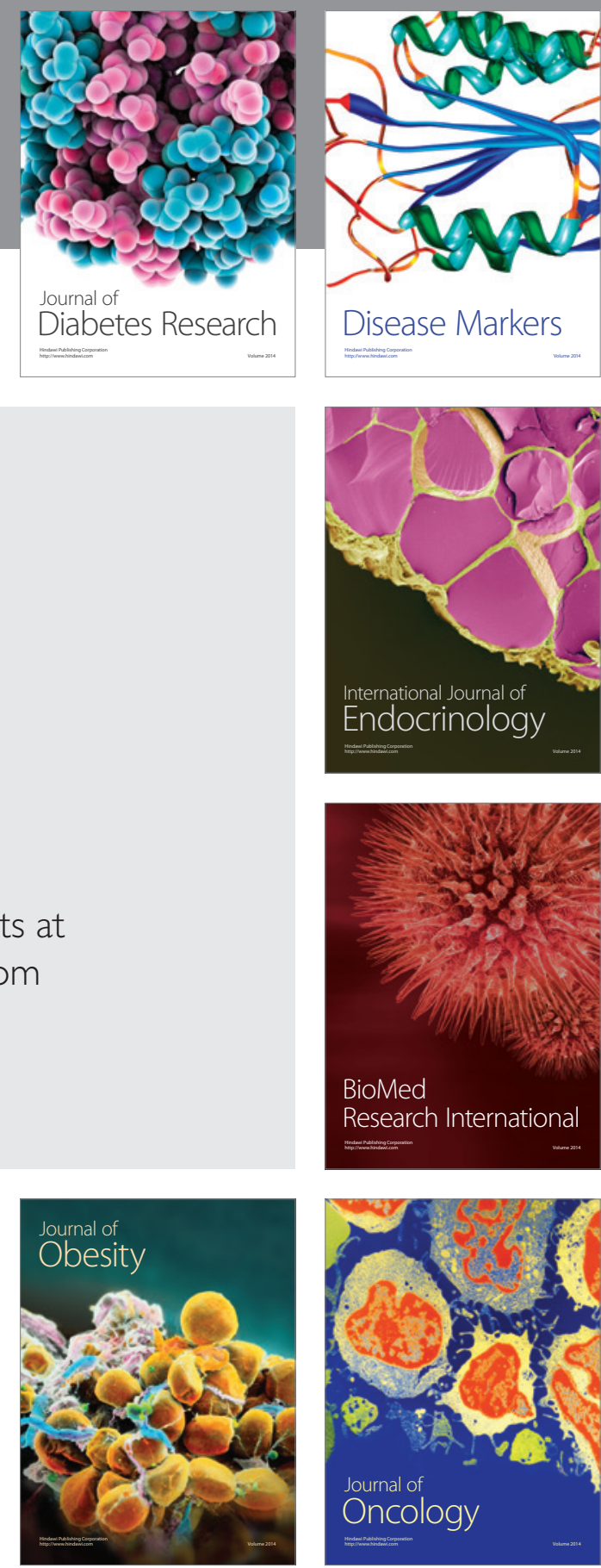

Disease Markers
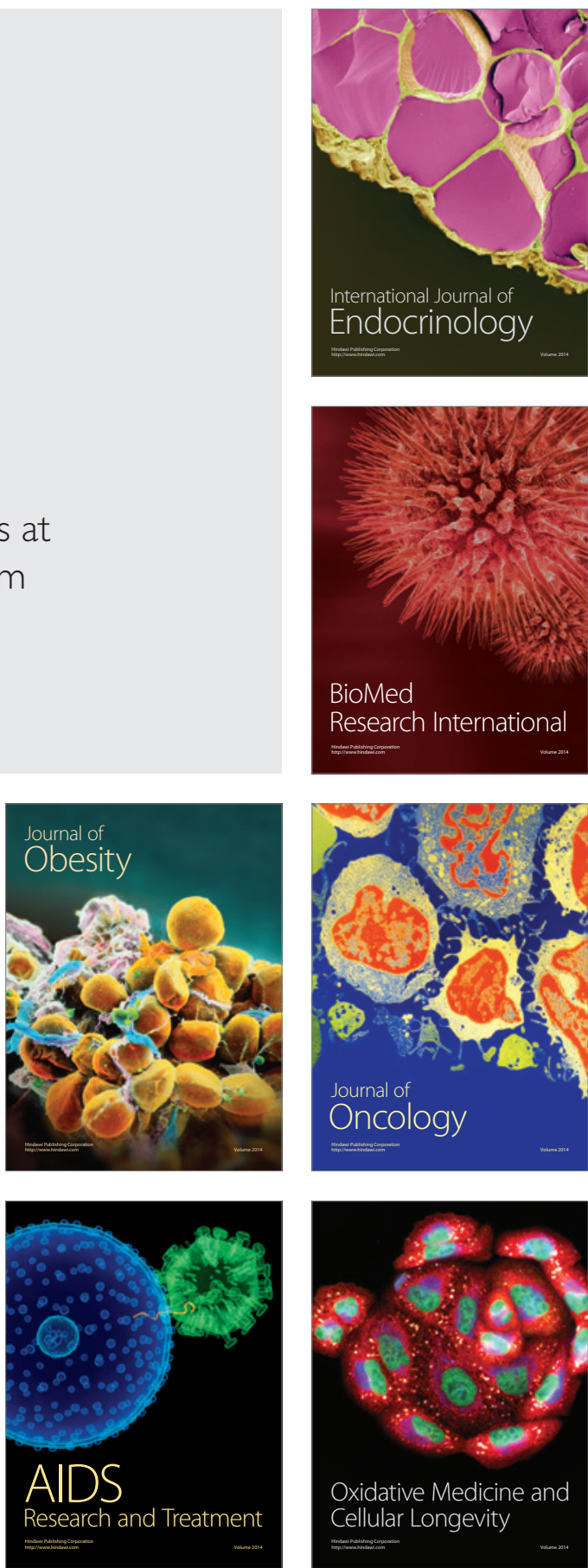